\title{
Do People Exploit Their Bargaining Power? An Experimental Study*
}

\author{
Ken Binmore, Peter Morgan, Avner Shaked, and John Sutton
}

Economics Department, University of Michigan, Ann Arbor, Michigan 48109

Received June 30, 1989

\begin{abstract}
Do people "play fair," or do they exploit their bargaining power? This paper suggests that such questions may not be well posed. It studies two bargaining situations that are very similar from the point of view of current fairness/focal theories of behavior, but differ in their strategic aspects. Different behavior in the two cases and a tendency by subjects after the experiment to describe as "fair" what actually occurred are reported. Journal of Economic Literature Classification Numbers: 026, 215. () 1991 Academic Press. Inc.
\end{abstract}

\section{INTRODUCTION}

Human behavior, even in simple bargaining situations, is not well understood. Numerous rival theories compete for attention, but the data are seldom adequate to justify a rejection of one in favor of another. It is often not even clear what the significant control variables are.

This paper examines only one small aspect of the problem. It describes an experimental attempt to compare the predictions of two qualitatively distinct types of theory. First, there are the fairness/focal theories of bargaining behavior as propounded by Güth (1990), Kahncman et al. (1986), Roth (1985), Selten (1978), and others. Second, there are the strategic theories of bargaining behavior, notably that of Rubinstein (1982). Game-theoretic or strategic models treat the bargainers as rational optimi-

\footnotetext{
* We are grateful to the Economics and Social Research Council of the United Kingdom for generously funding this research project. We also thank P. Knox and S. Chew for efficiently programming the experiments, and A. Hoolighan, A. Klin, C. Mirrlees, C. Purkhardt, and B. Thakker for their invaluable help in supervising the experiment and recruiting the subjects. We are also grateful to the Psychology Department at the London School of Economics for the use of their laboratory.
} 
zers and hence predict that the players will exploit whatever bargaining power they possess. Fairness/focal theories view the agreement on which subjects settle as being determined by social norms or conventional understandings that render the agreement focal, given the circumstances in which the bargainers are working. In a bargaining context, the social normals often involve "fairness" considerations, but other features of the siluation may also be important. For example, deals involving whole numbers of dollars may be salient in some circumstances.

It is not easy to distinguish fairness/focal behavior from strategic behavior. Indeed, part of the message of this paper is that what people perceive as "fair" or focal can sometimes be explained in terms of the strategic realities of the situation. However, we found it possible to design two simple laboratory games that, superficially at least, seem very similar from a fairness/focal viewpoint but which differ significantly in their strategic characteristics.

To summarize the results of the experiments very briefly, the subjects' behavior was biased in the direction of strategically optimal play. Under one of the two conditions, the differences in behavior between the two types of game were very marked indeed. The same turned out to be true of what the subjects asserted to be fair when questioned on this issue after playing the game. ${ }^{\text {T }}$ That is to say, what they judged to be fair after experiencing actual play was biased in the direction of the outcome that would result from strategically optimal behavior in the game they had actually played.

Peter Cramton (1988) has run the same experiment using our computer programs with Yale undergraduates as his subjects. His conclusions will be reported elsewhere. We note only that they are broadly consistent with ours. However, his subject population was half the size of ours.

Commentary on the results is left to a concluding section. At this point, we observe only that the fairness/focal and strategic bargaining literatures by no means exhaust all possible viewpoints. See, for example, Leventhal (1980), Thibaut (1968), or Walster et al. (1973).

\footnotetext{
${ }^{1}$ Individuals in the same pool from which the subjects were drawn but who did not play the game were also surveyed on the "fairness" question. There was no significant difference on what was reported as "fair" in the two types of game. This fact could be used for rhetorical purposes in support of the conclusions of the paper, but we do not feel the very dispersed data are good enough for this purpose. In asking inexperienced people for an opinion about a complicated matter, one must expect noisy answers: and it may well be that the data from the poll contain essentially nothing but noise with little or no relevance to the experiment. The best that one would seem entitled to conclude is that the description of the two types of game offered to the subjects for an opinion did not trigger any firmly held preconceptions about what is or is not fair in bargaining situations.
} 


\section{Bargaining Models}

The basic problem for the subjects in all the models considered is that of dividing a sum of money ${ }^{2}$ that we call a "cake." If the negotiations break down, player I will receive a payment which is equivalent to a share $\alpha$ of the cake and player II will receive a share $\beta$ of the cake, where $\alpha+\beta \leq 1$. What is fair in such a situation? Three possible answers to this question merit special attention:

(a) Split-the-difference (S-T-D). With this outcome, player I is assigned $\alpha$, player II is assigned $\beta$, and then they split the remainder of the cake equally. This outcome is the Nash bargaining solution with the status quo located at $(\alpha, \beta)$. Player I's final share is then $(1+\alpha-\beta) / 2$ and player II's share is $(1+\beta-\alpha) / 2$.

(b) Fifty-fifty (50:50). With this outcome, the breakdown payments $\alpha$ and $\beta$ are ignored and each player simply gets half the cake.

(c) Deal-me-out (D-M-O). With this outcome, the breakdown payments are ignored and the result is $50: 50$, unless this would assign player $i$ less than $i$ 's breakdown payoff of $\gamma$. If so, player $i$ gets $\gamma$ and the other player gets the remaining $1-\gamma$ of the cake.

The term deal-me-out derives from a previous paper (Binmore et al., $1989 \mathrm{c})$ and is intended to suggest player $i$ 's response to the proposed implementation of 50:50 when $\gamma>\frac{1}{2}$. Its possible role as a "fairness" criterion was suggested by critics of the previous paper.

In all the games considered, $\alpha$ was taken to be very small $(\alpha=0.04)$. Two values of $\beta$ were considered: a high value $(\beta=0.64)$ and a low value $(\beta=0.36)$. Figure 1 provides a convenient means of comparing the three different notions for different values of $\beta$ (but with $\alpha$ fixed at 0.04 ).

To discuss strategically optimal play, it is necessary to be specific about the bargaining procedure to be used. We employ a procedure studied by Rubinstein (1982). Accessible accounts of variants of his model, including those considered here, are to be found, for example, in Binmore et al. (1989b) or Sutton (1986).

In the Rubinstein procedure, the players alternate in making proposals indefinitely until a proposal is accepted or the negotiations break down. Some incentive is necessary to encourage the players to reach an early agreement. The two classes of games considered differ in how this incentive is provided and in how breakdowns may occur.

\footnotetext{
${ }^{2}$ We proceed throughout on the questionable assumption that utility can be identified with money.
} 


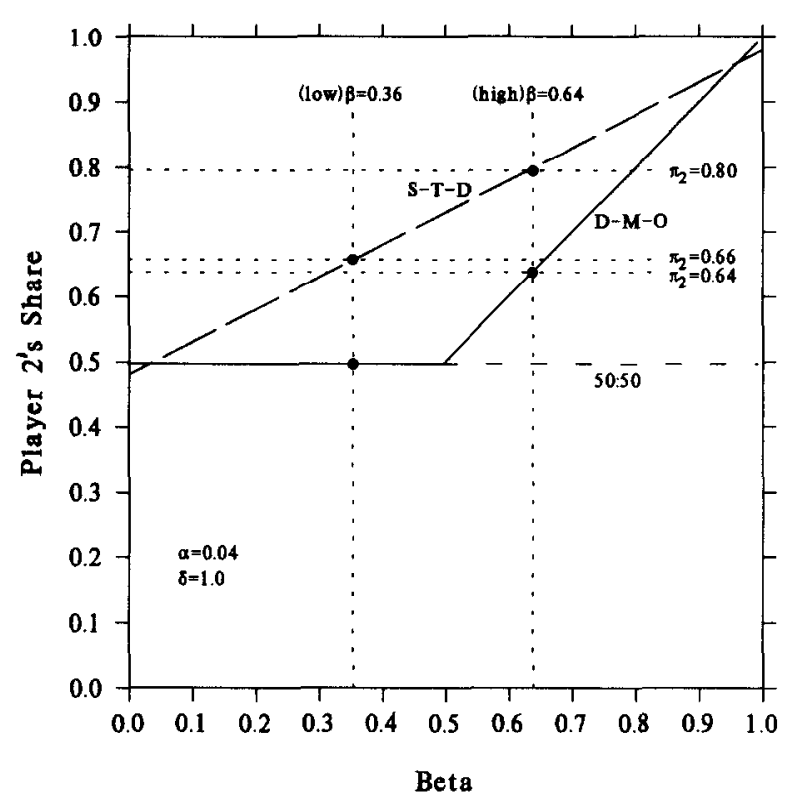

FIG. 1. The "fairness" criteria.

(A) Games with optional breakdown. In these games, a player may opt out after refusing a proposal ${ }^{3}$ made by the opponent (and only then). If a player opts out, the negotiations are deemed to have broken down, and the players receive their breakdown shares, $\alpha$ and $\beta$, of the current cake. The incentive for an early agreement is provided by the fact that the cake and the outside options shrink by a factor of $\delta$ immediately before each proposal after the first.

The game has a unique subgame-perfect equilibrium in which player I offers a share equal to $\max \{\beta, \delta /(1+\delta)\}$ to player II at time 0 , and player II accepts (Binmore et al., 1989c). Note that the equilibrium outcome converges to D-M-O as $\delta \rightarrow 1-$.

(B) Games with forced breakdown. In these games, players may not choose to opt out and the cake does not shrink. Instead, after each refusal, a random move decides whether the negotiations will be broken off or allowed to continue. The probability of a continuation is taken to be the same value of $\delta$ as in optional breakdown games. The incentive for early agreement is therefore that the cake may disappear altogether if the negotiations are prolonged, leaving each player with only their breakdown payment.

\footnotetext{
${ }^{3}$ It matters when a player may opt out (Shaked. 1987).
} 
TABLE I

\begin{tabular}{lcc}
\hline & $\beta=0.36$ & $\beta=0.64$ \\
\hline Optional breakdown & Regime 0 & Regime I \\
& Low optional & High optional \\
Forced breakdown & Regime 2 & Regime 3 \\
& Low forced & High forced \\
\hline
\end{tabular}

The game has a unique subgame-perfect equilibrium in which player 1 offers a share equal to $\{\delta(1-\alpha)+\beta\} /(1+\delta)$ to player II at time 0 , and player II accepts (Binmore et al. 1989c). Note that the equilibrium outcome converges to S-T-D at $\delta \rightarrow 1-$.

Each of these two classes of games was run under two conditions: low $\beta$ and high $\beta$. This yields four different games that are referred to as regimes $0,1,2$, and 3 as indicated in Table I. Thus the labels 0 and 1 refer to optional breakdown games with low and high $\beta$, respectively, and the labels 2 and 3 refer to forced breakdown games with low and high $\beta$, respectively.

Two points should be noted. The first is that a subgame-perfect analysis predicts future behavior even if past behavior has not been as predicted. Our earlier work (Binmore et al., 1989c) on optional breakdown games indicates that one should not expect instant agreement at time zero from subjects in the laboratory, but that there is reason to believe that the game-theoretic prediction of the final outcome, in terms of the cake then available, may not fare too badly. Results are therefore always reported in terms of player II's share of the cake available at the time the game ended. When $\delta \rightarrow 1-$, the game-theoretic prediction will always be D-M$\mathrm{O}$ in optional breakdown games, and S-T-D in forced breakdown games. The second point is the more important. For a given value of $\beta$, optional breakdown games and forced breakdown games are intended to present a similar payoff profile to the subject, who may therefore be inclined to treat them in the same way in deciding what is or is not fair or focal. Indeed, since a subgame-perfect analysis of an optional breakdown game is identical to that of a forced breakdown game when $\alpha=\beta=0$, one might expect even a strategically minded but inexperienced subject to fail to recognize the rather subtle distinction between the two classes of game. If subjects behave differently in optional breakdown games from the way they behave in forced breakdown games, one would therefore seem to have evidence in favor of the players' bargaining power being a significant factor in determining the final outcome. It is this consideration that provides the major motivation for the experimental design described in this section. 


\section{THE EXPERIMENT}

Subjects were recruited directly from undergraduate classes in economics at the London School of Economics. The students had not studied game theory or bargaining, nor were these topics part of the curriculum for the courses they were attending. The recruiters were graduate students in Psychology who also supervised the fully automated experimental runs in the Psychology Laboratory. Subjects were informed that the experiment was "in economics" rather than "in psychology," but were not informed of the identities of the authors of this paper. As to the details of the game itself, our intention was that the subjects be perfectly informed about the rules of the game and the monetary payoffs to be distributed.

The main experiment ran for 6 days. Each day had four sessions, with each session devoted to a different regime from Table I. Each session involved four subjects, who each played 10 games in all. After each game, a subject's opponent was changed. On arrival, subjects were seated in isolated booths with a minimum of interaction between them. They communicated via networked microcomputers. They were first asked to read written instructions (Appendix A), and then to operate a demonstration program that provided them with hands-on experience on how to make and respond to offers and so on. The cake was represented on the screen by a rectangular slab. The subject made an offer by moving a "knife" up or down the cake until satisfied with the division it indicated. The monetary amounts being proposed were also displayed. The demonstration program did not involve any examples of partitions of the cake since we were anxious not to interfere with the natural focal point structure of the game.

After running the demonstration program and asking any questions they might have, ${ }^{4}$ the subjects then played six "practice games" for which no payments were made. They then played four "real games" in each of which the cake was initially worth $£ 5.00$. At the time of the experiment (December 1987), this was worth about $\$ 8.00$. We felt this sum provided an adequate incentive for the subject to devote some care and attention to the experiment, given that we were asking for only 30-45 min of their time. Since each group of four subjects in a particular session played a total of 20 games altogether (12 for practice and 8 for real) and since each regime was in force on each of 6 days, we observed a total of 120 games for each of the four regimes ( 72 for practice and 48 for real).

To minimize on reputation effects, the subjects' bargaining partners were changed after each game. Their role in the game also varied. Half the

\footnotetext{
${ }^{4}$ A supervisor could be summoned by pressing an appropriate key.
} 
time they occupied the role of player I (who moves first and has a breakdown payment of $\alpha$ ) and half the time they occupied the role of player II (who moves second and has a breakdown payoff of $\beta$ ). We attach importance to this alternation of roles in game-theoretic experiments. A rational player bases his strategic analysis of a game on the way he would play if he were in the shoes of the opponent. Alternating roles provides subjects with an opportunity to see things from the other player's viewpoint and hence to understand the game better. In a previous experiment (Binmore et al., 1985, 1988), such role switching influenced the outcome very markedly.

At the end of each session, subjects were asked to remain seated until they had completed a questionnaire (Appendix B) and had been paid the total amount of money they had successfully bargained for in the four real games that each had played. They were then invited to leave one by one with a view to minimizing interaction.

Under all the four regimes of Table 1 , the cake was worth $£ 5.00$ in the main experiment and the parameter $\delta$ was taken to be 0.9 . In all four regimes, player I's breakdown share of $\alpha=0.04$ was therefore initially worth $£ 0.20$ in money.

For optional breakdown games (regimes 0 and 1), the cake shrinks over time according to the discount factor $\delta$, and it is left to the players' discretion whether to force a breakdown by opting out. Under regime 0 , player II's breakdown share of $\beta=0.36$ was initially worth $£ 1.80$. Under regime 1 , player II's breakdown share of $\beta=0.64$ was initially worth $£ 3.20$.

For forced breakdown games (regimes 2 and 3), the cake does not shrink, but there is a risk of an imposed breakdown every time that an offer is refused. Our intention was that the players should believe that the game continues after a refusal with probability $\delta=0.9$, but here we met with a difficulty in our pilot experiments. The manner in which we sought to resolve this difficulty requires some explanation.

In our initial pilot, the written instructions described the probabilistic mechanism by means of which breakdown occurred and, after each refusal, subjects saw a simulated roulette wheel turn on their screens. Nevertheless, they tended to behave as though the possibility of a breakdown ever occurring was negligible. ${ }^{5}$ That is to say, they neglected to note that, although 0.9 is nearly $1,(0.9)^{n}$ is small when $n$ is sufficiently large. Such misconceptions about probabilistic matters are, of course, commonplace as laboratory phenomena.

After various attempts, we sought to evade the difficulty by telling the

${ }^{5}$ And, in many cases, confirmed this interpretation of their behavior by their comments on the questionnaire. 
subjects, in their written instructions, that the maximum length for each game had already been chosen in advance, but that they were not to be told what this length was. However, they were invited to proceed on the assumption that, after each refusal, the probability of the game continuing was 0.9 . The precise wording was as follows:

In each of the ten sessions, the number of proposals allowed before a breakdown is announced has been fixed in advance. But we are going to keep you guessing by not telling you what these ten numbers are. All you will know for sure is that a breakdown will occur eventually if agreement is delayed long enough. The maximum number of proposals allowed in each session may be large or it may be small, and knowing what the number turns out to be in one session will not help much in guessing what it will be in another. The numbers have been fixed so that, however many proposals there may already have been in a session, you should still reckon that there is a $90 \%$ chance of being allowed at least one more proposal. This means for example, that it is more likely that 12 or more proposals will be allowed than 3 or less.

No subject expressed any confusion about the issue on their questionnaire. We chose the maximum lengths for 10 games that each subject played to be:

$$
9,2,11,2,10,7,7,16,12,8 \text {. }
$$

The two short games were intended to convince the subjects that breakdown could indeed occur. Otherwise, our intention was that the data available to the subjects should not be such as to allow them rationally to reject the hypothesis that breakdowns occur independently with probability 0.1 , even if they participated in games that always ended in breakdown. ${ }^{6}$

\section{Results}

The raw results are available as an appendix to a discussion paper Binmore et al. (1989a). We will be pleased to supply a copy of this discussion paper on request. In this section, the results we believe to be relevant are summarized in six histograms (Fig. 2-4). We always report percentages of the cake obtained by player II. (In optional breakdown games, the cake shrinks over time. The percentage of the cake is then computed in terms of the cake available at the time the game ended.) Games that do not end in agreement are indicated by an empty box. Player II then gets

${ }^{6}$ We do not, of course, believe that the subjects did carry out any elaborate probabilistic calculations. It is enough for our purposes if the subjects are convinced that the game will end eventually but that it is unlikely to do so immediately. 
his or her breakdown share $\beta$ (36\% under regimes 0 and $2 ; 64 \%$ under regimes 1 and 3).

The immediate issue is whether, in view of the similarity of their payoff profiles and the subtlety of their strategic differences, optional breakdown and forced breakdown games generate the same behavior, although a subgame-perfect analysis predicts D-M-O in the former and S-T-D in the latter. Before considering this question, some preliminary comments are useful:

\section{How Big is Epsilon?}

Game theory treats players as rational optimizers, who are assumed to squeezc the last penny from a situation on the assumption that their opponent will do the same. But in practice, one must accept that subjects will treat small enough amounts as negligible. As a rule-of-thumb, we proceed as though anything less than the price of a cup of coffee $(£ 0.2=$ $4 \%$ of $£ 5.00$ at the time of the experiment) is negligible. In particular, we neglect the fact that the strategically optimal outcomes with the actual discount factor used $(\delta=0.9)$ differ slightly ${ }^{7}$ from those in the limiting case when $\delta \rightarrow 1-$.

\section{Round Number Focal Points}

A further source of possible distortion is the tendency of subjects to settle on deals in round numbers. Under the high $\beta$ regimes 1 and 3 , this tendency makes S-T-D attractive (since $80 \%$ of the cake is $£ 4.00$ at time 0 ) and creates a possible focal point at $70 \%$ (which corresponds to $£ 3.50$ at time 0 ). Under the low $\beta$ regimes 0 and 2 , a round number focal point may exist at $60 \%$ (which corresponds to $£ 3.00$ at time 0 ).

\section{Disagreements}

It is sometimes argued that the fact that subjects often fail to agree immediately in bargaining games of perfect information is a serious obstacle to a game-theoretic interpretation of their behavior. It is true that, in the models of this paper, all equilibrium offers will be accepted in equilibrium and hence any refusal is an out-of-equilibrium phenomenon. However, although he accepts in equilibrium, the responder in these models is always indifferent between accepting and refusing. Thus a refusal by a responder does not necessarily represent a large deviation from the game-

\footnotetext{
${ }^{7}$ For example, under regime 0 , a subgame-perfect analysis predicts $47.42 \%$ for player II when player I makes the first offer, and $52.67 \%$ when player II makes the first offer. These are both approximated by the $50 \%$ predicted by D-M-O.
} 
theoretic prcdiction if deviations are measured in terms of the responder's anticipated payoffs. ${ }^{8}$

This discussion is particularly relevant to disagreements in the "high optional" games of regime 1. A subgame-perfect equilibrium analysis of such games predicts that player I will offer player II approximately $\beta=$ $64 \%$ at time 0 , and that this will be accepted. But such an offer is approximately equal to player II's outside option in such games. If he refuses the offer made to him and instead takes his outside option, it is true that he deviates from what the analysis predicts, but the deviation is negligible in terms of the payoff he receives. For this reason we count disagreements under the high optional regime 1 as being supportive of the game-theoretic prediction (namely D-M-O) rather than dismiss them as "noise."

We do not do the same for "forced breakdown" games. The bargaining in the games of this type that the subjects played for money was unilaterally terminated by the computer after a minimum of seven offers had been rejected. Disagreements in forced breakdown games therefore convey little information relevant to this study, beyond the fact that disagreements do indeed occur even though they are not predicted. ${ }^{9}$ The same goes for the "low optional" games of regime 0 .

\section{The Data}

A statistical analysis appears in Section 7 . Figure 2 summarizes the data for the high $\beta$ regimes 1 and 3. The difference of behavior between optional breakdown and forced breakdown games is very marked. At this point we note only that game theory predicts the observed behavior much better than the fairness/focal alternatives listed in Section 2 that take no account of strategic issues. It is not surprising that $50: 50$ does not do well when player II can get $64 \%$ without the consent of his partner, ${ }^{10}$ but it is instructive that S-T-D predicts very much better than D-M-O in forced breakdown games, while D-M-O predicts better than S-T-D in optional breakdown games.

The results for the low $\beta$ regimes 0 and 2 are summarized in Fig. 3. Here the differences between optional breakdown and forced breakdown are

\footnotetext{
${ }^{8}$ Moreover, a prediction based on a subgame-perfect equilibrium analysis presupposes that it is common knowledge that the players are "perfectly rational." If subjects entertain doubts on this score, one might also see deviations from equilibrium by proposers who are "testing the rationality" of their opponent. One might then also observe deviations by responders who anticipate such behavior from their opponent in the future. This latter point is relevant to what follows in the text on opting out.

"Note in particular that breakdown in the "high forced" regime 3 is not compatible with D-M-O when the latter is regarded as a "fairness" criterion because, although player II gets $\beta$, player I only gets $\alpha$ instead of his "fair" share of $1-\beta$.

${ }^{10}$ Although a number of researchers have observed systematic violations of individual rationality in related contexts.
} 
Regime 1: High-Optional

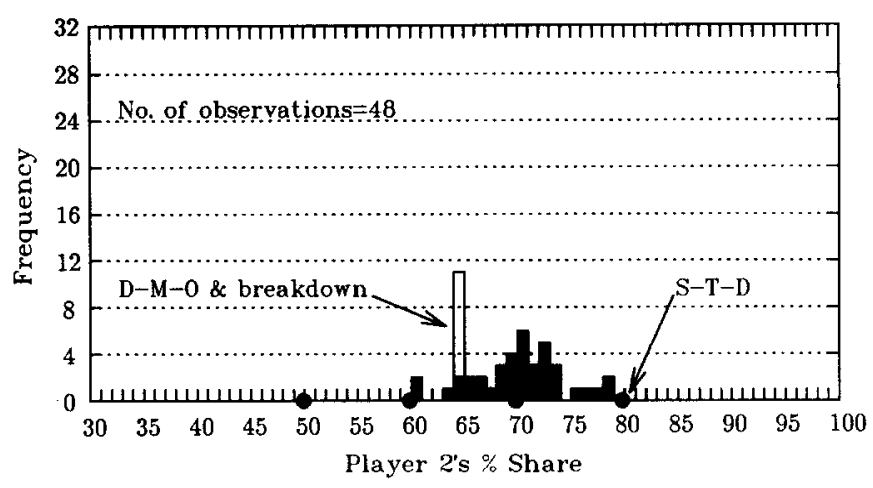

Regime 3: High-Forced

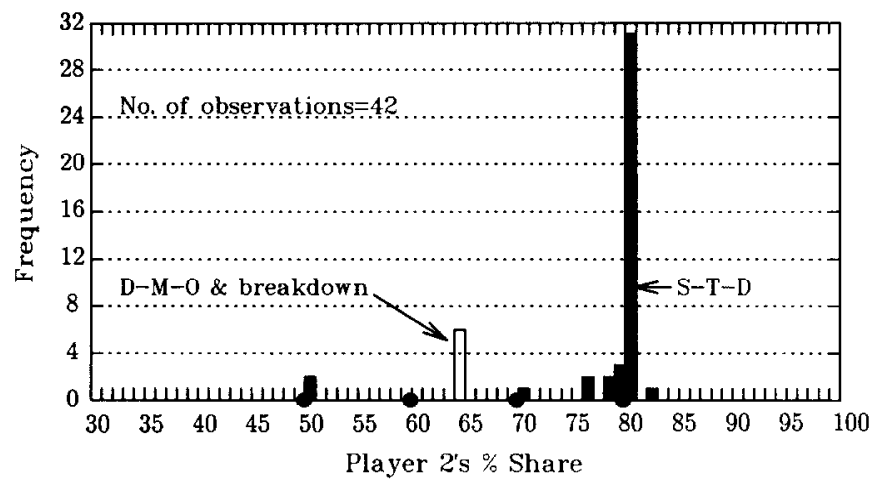

FIG. 2. A comparison of the paid "high optional" games with the paid "high forced" games for the $f 5$ cake. An open square $(\square)$ refers to the games that ended in a breakdown, and a solid square ( $\square$ ) to those that did not. The solid circles $(\bullet)$ on the horizontal axis at 50 , 60,70 , and $80 \%$ indicate possible round number focal points. These percentages of $£ 5$ are $£ 2.50, £ 3.00, £ 3.50$, and $£ 4.00$, respectively.

slight. The round number focal point at $60 \%$ ( $£ 3.00$ at time 0 ) is perhaps responsible for producing this result, since it lies roughly midway between D-M-O (50\%) and S-T-D (66\%).

To examine this possibility, we ran the low $\beta$ regimes 0 and 2 again, but with the $£ 5.00$ cake replaced by an $\$ 11.00$ cake. Subjects were told that their dollar winnings would be paid to them in pounds sterling at the then current exchange rate. Otherwise the circumstances of the experiment were identical. The point of doing this was to create two round number focal points (at $\$ 6.00$ and $\$ 7.00$ ) between the D-M-O prediction of $50 \%$ and the S-T-D prediction of $66 \%$. Figure 4 shows the sharper data obtained. The differences between optional breakdown and forced breakdown games are statistically significant (Section 7). Game theory cannot 


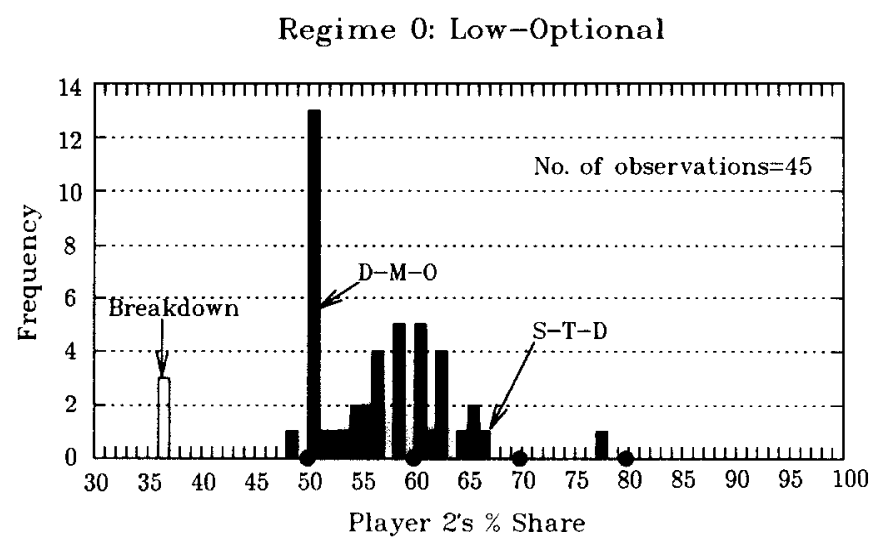

Regime 2: Low-Forced

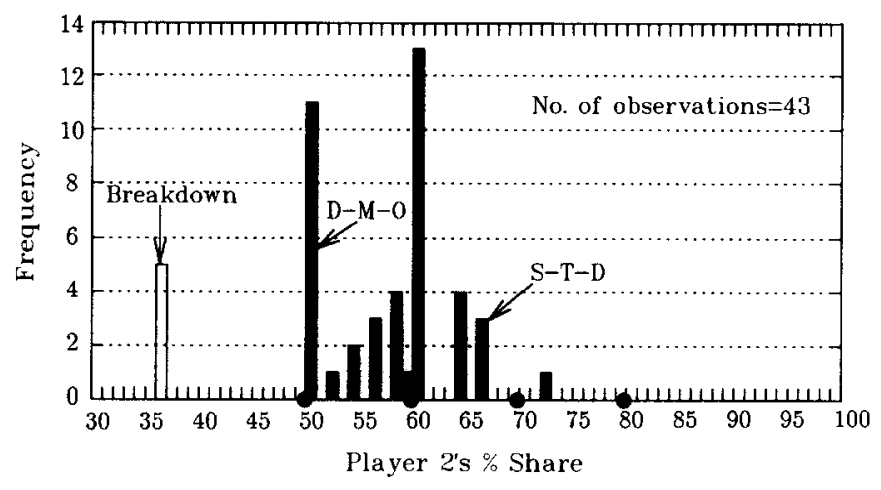

FIG. 3. A comparison of the paid "low optional" games with the paid "low forced" games for the 15 cake. An open square $(\square)$ refers to the games that ended in a breakdown, and a solid square $(\boldsymbol{D})$ to those that did not. The solid circles $(\boldsymbol{\theta})$ on the horizontal axis at 50 , 60,70 , and $80 \%$ indicate possible round number focal points. These percentages of $£ 5$ are $£ 2.50, £ 3.00, £ 3.50$, and $£ 4.00$, respectively.

be said to predict these data well, but it does better than the fairness/focal alternatives being considered.

\section{QUESTIONNAIRE}

Interesting results were obtained from the subjects' answers to the questionnaire (Appendix B). We discuss only ${ }^{11}$ the answers to Questions

"It is not clear to us how much weight can be given to the answers to Question 8. For the record, we observe that $50 \%$ of the subjects were unambiguously of the view that it is socially acceptable to use one's bargaining power, and $17 \%$ were unambiguously of the opinion that one ought to "play fair." 
Regime 0: Low Optional

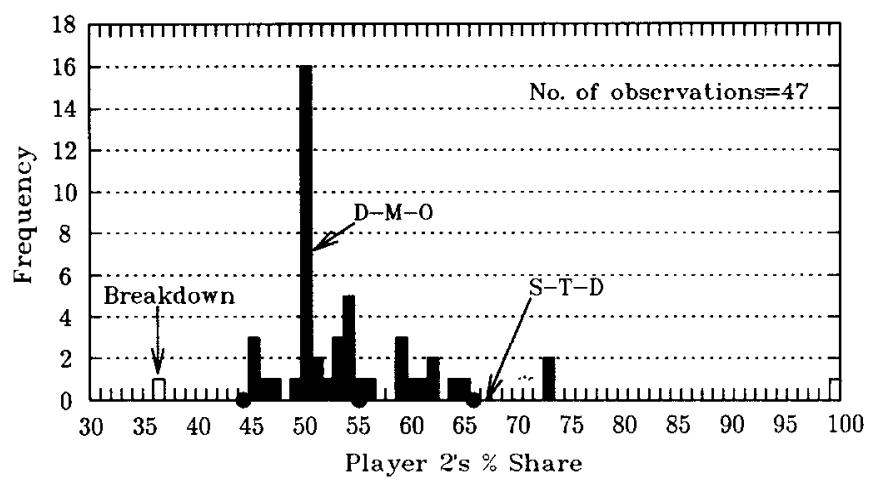

Regime 2: Low-Forced

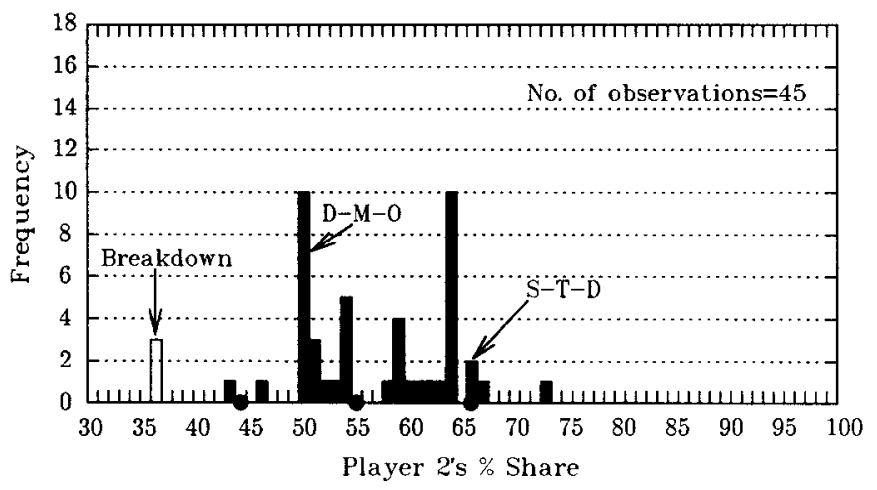

FIG. 4. A comparison of the paid "low optional" games with the paid "high optional" games for the $\$ 11$ cake. An open square ( $\square$ ) refers to the games that ended in a breakdown, and a solid square (D) to those that did not. The solid circles $(\bullet)$ on the horizontal axis at 45 , $55,64 \%$ indicate possible round number focal points. These percentages of $\$ 11$ are $\$ 5.00$, $\$ 6.00$, and $\$ 7.00$, respectively.

5,6 , and 7 . In these questions, the subjects were asked to indicate what they felt to be a fair way to split the cake in three situations. In each situation, player I's breakdown share of the cake was $\alpha=0.04$ but player II's was successively $\beta=0.04, \beta=0.36$, and $\beta=0.64$. The unanimous response to Question $5(\alpha=\beta=0.04)$ was that $50: 50$ is fair in this symmetric situation.

The answers to Question $6(\beta=0.36)$ and Question $7(\beta=0.64)$ are summarized in Figs. 5 and 6 . Figure 5 shows the views about fairness expressed by all those who had experienced optional breakdown games 


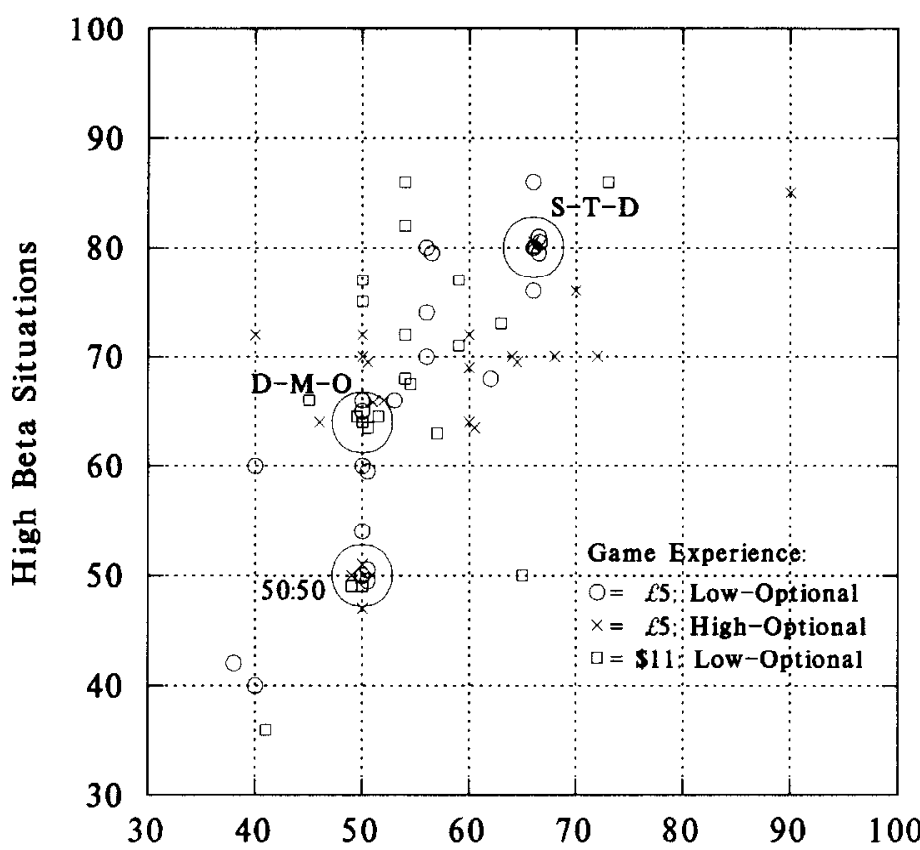

\section{Low Beta Situations}

FIG. 5. The share of the cake for player II proposed as "fair" for low and high $\beta$ situations by subjects who had experienced optional breakdown games.

(in which D-M-O is strategically optimal). The $x$-coordinate of a point ${ }^{12}$ in the figure shows what was asserted to be fair in a low breakdown payoff situation $(\beta=36 \%)$ and the $y$-coordinate shows what was asserted to be fair in a high breakdown payoff situation $(\beta=64 \%)$. Figure 6 similarly shows the views of all those who had experienced forced breakdown games (in which S-T-D is strategically optimal).

The difference between Figs. 5 and 6 is striking. (See Section 7 for a statistical analysis.) Note in particular the following features:

1. In both figures, a small group (around 10\%) insists that 50:50 is "fair" in spite of the asymmetries they are invited to contemplate.

2. Those who had experienced forced breakdown games (S-T-D strategically optimal) were very much more in agreement about what is fair

${ }^{12}$ Too much significance should not be attached to the precise location of points in Fig. 5 and 6 . For example, in Fig. 6, most subjects simply proposed the S-T-D point. These choices have been indicated by clustering them as close to the S-T-D point as possible without overlaps. Also, subjects were not always very neat in marking their choice of a "fair division" on their questionnaires. 


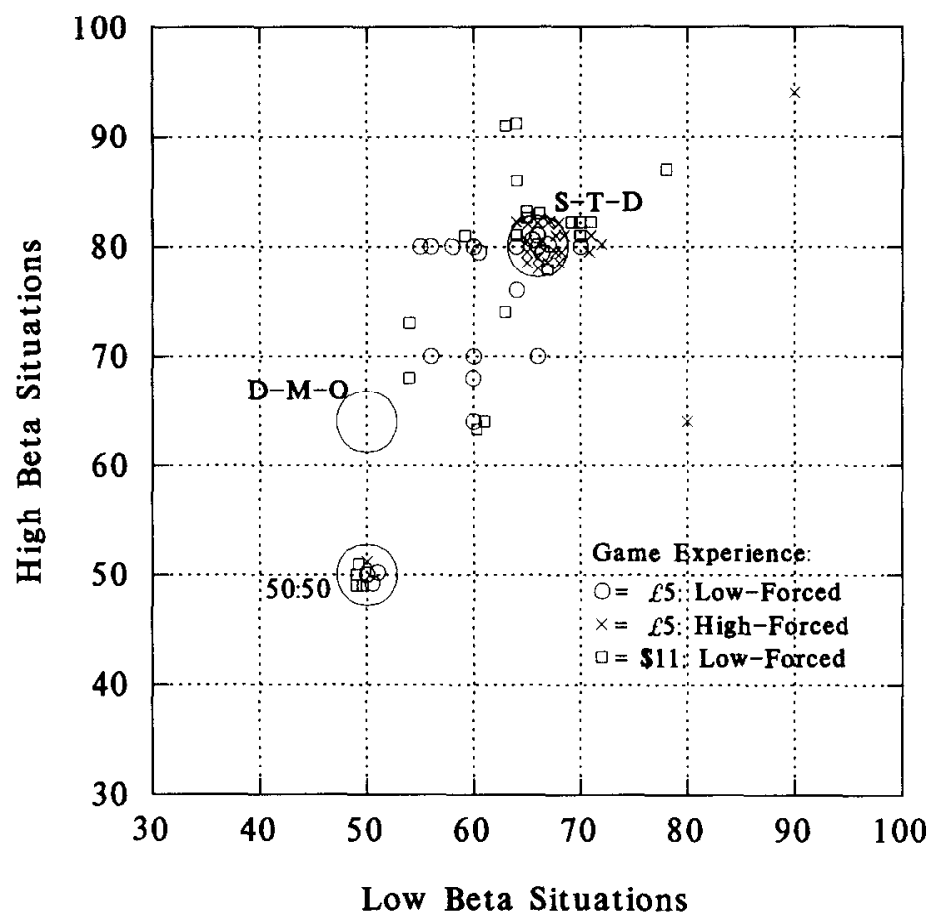

FIG. 6. The share of the cake for player II proposed as "fair" for low and high $\beta$ situations by subjects who had experienced forced breakdown games.

than those who had experienced optional breakdown games (D-M-O) strategically optimal). Results for optional breakdown games were very much more dispersed.

3. In forced breakdown games, S-T-D predicts what was asserted to be fair quite well, and D-M-O predicts very badly.

4. In optional breakdown games, the situation is more confused. However, D-M-O is no longer irrelevant to the data.

We do not feel that these results are conclusive, but they do suggest that people's views about what is fair may be heavily influenced by their strategic experiences in situations about which they do not have established preconceptions.

\section{UNLEARNING}

We were disappointed not to have sharper results under the optional breakdown regimes 0 and 1 , since we had obtained sharp results in a 


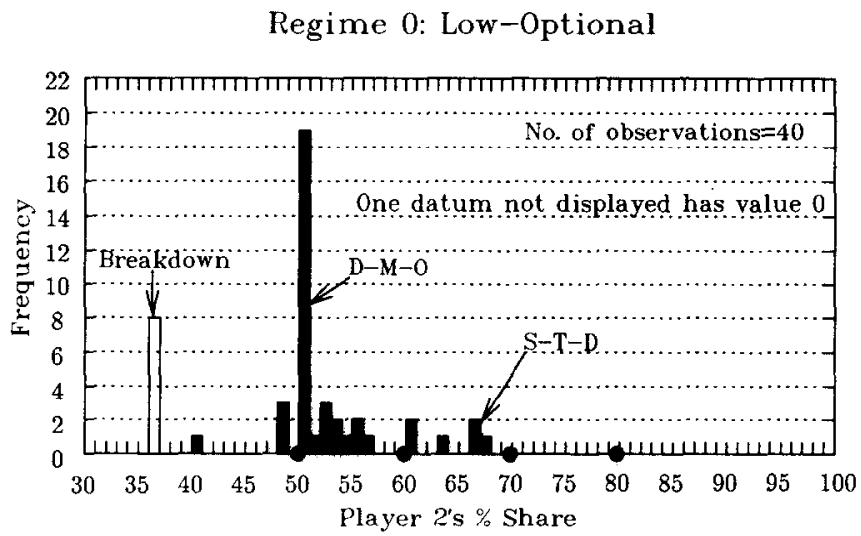

Regime 1: High-Optional

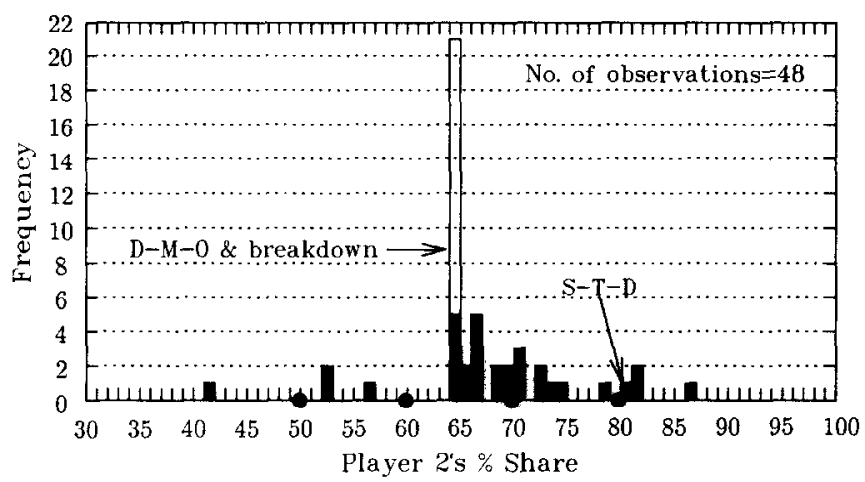

FIG. 7. The first four practice games with optional breakdown for both low and high $\beta$ in the case of the $£ 5$ cake. A open square $(\square)$ refers to the games that ended in a breakdown, and a solid square ( $\square)$ to those that did not. The solid circles $(\bullet)$ on the horizontal axis at 50 , 60,70 , and $80 \%$ indicate possible round number focal points. These percentages of $£ 5$ are $£ 2.50, £ 3.00, £ 3.50$, and $£ 4.00$, respectively.

previous study ${ }^{13}$ of optional breakdown games (Binmore et al., 1989c), without apparent interference from round number focal points. However, in this previous study, subjects did not play repeatedly and hence had little opportunity to learn. It is therefore of interest to compare the results of our previous study with those shown in Fig. 7 for the first four practice games under the optional breakdown regimes 0 and 1 in the case of the f5.00 cake. $^{14}$

13 The size of the cake and the values of $\alpha$ and $\beta$ were not the same.

${ }_{14}$ We did not run regime 1 with an $\$ 11$ cake. The results from practice games under regime 0 for the $\$ 11$ cake are very similar to those for the $\$ 5$ case. Only the first four practice games are reported so that the number of observations in each of the histograms of Fig. 2, 3, 4, and 7 is the same. 
The results from these practice games and those from our previous study are similar, in that D-M-O predicts the data quite well in absolute terms, and overwhelmingly better than S-T-D. The drift away from this distribution is evident from Fig. 3. One can tell a story for the high $\beta$ regime 1 about subjects learning that player II needs an epsilon on top of what is available from opting out if he or she is to be kept at the negotiating table. However, this would not seem to explain why player II's payoffs should improve over time under the low $\beta$ regime 0 . Presumably round number focal points are somehow relevant. Evidence in support of this would seem to be provided by the differing results oblained for the low $\beta$ regime 0 with an $\$ 11$ cake (Fig. 4). More research will perhaps provide an explanation for what is going on here. For the moment, the only safe conclusion would seem to be that, if people are indeed "natural gamesmen," 15 then experience in this context would appear to lead to some "unlearning" of their game-playing skills.

\section{Statistical Analysis}

Here we present the results of nonparametric tests of the null hypothesis that the data sets presented in Fig. 2, 3, 4, and 7 are generated, pair by pair, by the same stochastic process. A variety of tests suitable for testing this hypothesis exists. The most commonly used in this context seem to be the Kolomogorov-Smirnov (KS), Cramèr-von Mises (CM), and Anderson-Darling (AD) tests. Each compares empirical cumulative density functions (cdf's). A recent new test of Epps and Singleton (ES) (1986) compares empirical moment-generating functions.

The above tests are "nonparametric" in the sense that their significance levels are not affected by the actual stochastic process generating the observed data. However, the powers of the tests do depend upon the specifics of the two stochastic processes generating the data. Little is known about the finite sample size ranking of these tests by their powers for various types of stochastic processes. Consequently, we conducted a Monte Carlo study in an effort to deduce if one test or another might "dominate" the others by offering largest power in the context of our study. The results of this study are presented in Appendix $C$. The alternative distributions examined were selected so as approximately to mimic the empirical cdf's observed. We concluded that none of the four tests dominated the others, although the $\mathrm{CM}$ and $\mathrm{AD}$ tests generally performed at least as well as the KS and ES tests. These findings contrast with the results of the Monte Carlo study of Forsythe et al. (1988), who found that, as a pair, the $\mathrm{AD}$ and $\mathrm{ES}$ tests dominated the others. A number of reasons

${ }^{15} \mathrm{~A}$ view that has been wrongly attributed to us in the past. 
explain the differing conclusions. In particular, different sample sizes were used (25 in Forsythe et al. (1988) and 40 here) and different alternative hypotheses were examined.

The tests" results are presented in Table II. The "Value" columns report the observed values of the statistics. The "10\%" and "5\%" columns report if the tests retain the null hypothesis of no difference in distribution or reject it, at the $10 \%$ and $5 \%$ significance levels, respectively. The tests all reject the null hypothesis of no difference in the cases of Figs. 2 and 7, and all retain the null hypothesis for Fig. 3. The data for Fig. 4 lead to a strong rejection of the null hypothesis by the CM and AD tests, a rejection by the KS test at the $10 \%$ significance level, and a retention by the ES test (the prob-value of the ES test is $16 \cdot 5 \%$ ).

The two histograms of Fig. 4 suggest that the differences in the two data sets are, first, a shift in central location and, second, a difference in the size of a mass point. The Monte Carlo cases 2, 3, and 4 described in Appendix $\mathrm{C}$ investigate the relative powers of the four tests in the presence of shifts of central location and/or variance. Cases 8 and 9 were intended to reveal the tests's relative powers when a substantial mass point was introduced. In all of these cases the ES test is dominated (sometimes sharply) at either significance level by the CM or AD test. For these reasons we attribute more weight to the strong rejections of the null hypothesis of no difference by the CM and AD tests than to the somewhat weaker retention of the null hypothesis by the ES test.

We also tested the null hypothesis that the data presented in Fig. 5 and 6 were generated by the same bivariate process. There is a dearth of twodimensional nonparametric tests available for this task. Extensions of the $\mathrm{KS}$ and Wald-Wolfowitz tests from one dimension to two have been developed by Friedman and Rafsky (1979) but, as yet, are poorly tabulated. Accordingly, we adopted the following approach, which mimics techniques used by spatial statisticians. We categorized the data points into six regions by dividing the rectangle $[30,100] \times[30,100]$ vertically at $57 \cdot 5$ and horizontally at $57 \cdot 5$ and $71 \cdot 5$. The regions are labeled 1 to 6 from top left to bottom right. $57 \cdot 5$ is the horizontal coordinate halfway between the D-M-O (and 50:50) and S-T-D points. $71 \cdot 5$ is the vertical coordinate halfway between the D-M-O and S-T-D points. 57 is the vertical coordinate halfway between the D-M-O and $50: 50$ points. Any data points occurring on a boundary were assigned to the lower region. Doing so weakens the case for rejection of the null hypothesis. The respective numbers of data points in the regions are 10, 14, 20, 9, 12, and 1 for Fig. 5, a total of 66 data points, and 3, 48, 2, 6, 8, and 0 for Fig. 6, a total of 67 data points. The null hypothesis under test is that the two data sets are generated by the same stochastic process. Accordingly, we estimate the probability of a data point being generated for a particular region by combining the two samples and then computing the relative frequencies 


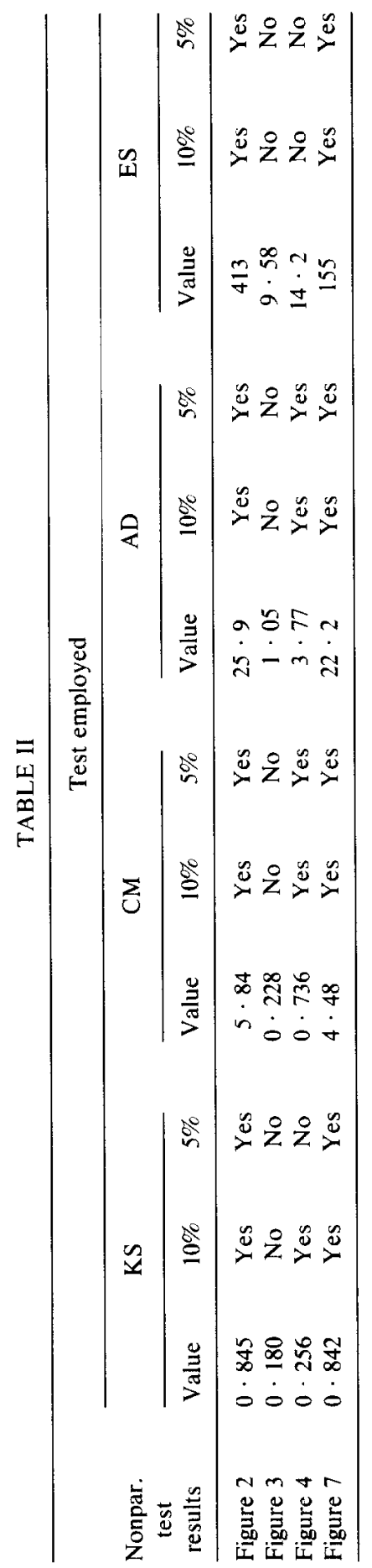


for the region from the combined sample of 133 data points. The respective probabilities so assigned to the six regions are $\frac{13}{133}, \frac{62}{133}, \frac{22}{133}, \frac{15}{133}, \frac{20}{133}$, and $\frac{1}{133}$. These probabilities imply that the marginal and conditional probabilities for a data point $\left(x_{1}, x_{2}\right)$ generated by the cstimated stochastic process are:

$$
\begin{gathered}
\operatorname{Pr}\left(30 \leq x_{1} \leq 57 \cdot 5\right)=\frac{55}{133}, \\
\operatorname{Pr}\left(57 \cdot 5<x_{1} \leq 100\right)=\frac{78}{133} \\
\operatorname{Pr}\left(30 \leq x_{2} \leq 57 \mid 30 \leq x_{1} \leq 57 \cdot 5\right)=\frac{20}{55}, \\
\quad \operatorname{Pr}\left(30 \leq x_{2} \leq 57 \mid 57 \cdot 5<x_{1} \leq 100\right)=\frac{1}{78} \\
\operatorname{Pr}\left(57<x_{2} \leq 71 \cdot 5 \mid 30 \leq x_{1} \leq 57 \cdot 5\right)=\frac{22}{55}, \\
\operatorname{Pr}\left(57<x_{2} \leq 71 \cdot 5 \mid 57 \cdot 5<x_{1} \leq 100\right)=\frac{15}{78}, \\
\operatorname{Pr}\left(71<x_{2} \leq 100 \mid 30 \leq x_{1} \leq 58\right)=\frac{13}{55}, \\
\operatorname{Pr}\left(71 \cdot 5<x_{2} \leq 100 \mid 57 \cdot 5<x_{1} \leq 100\right)=\frac{62}{78} .
\end{gathered}
$$

We used this bivariate stochastic process to generate two independent samples, the first of size 66 and the second of size 67 . The "distance" between the two samples was then computed as the sum, over the six regions, of the absolute values of the differences in the relative frequencies. We generated 1500 pairs of samples and computed the empirical distribution of the resulting 1500 values of the distance statistic. The range of the statistic is $[0,2]$ but the observed empirical distribution was concentrated almost cntircly between $0 \cdot 1$ and $0 \cdot 5$; the largest observed value was $0 \cdot 713$. The value of the statistic for the data displayed in Fig. 5 and 6 is $1 \cdot 009$. We conclude that the null hypothesis that the data presented in Fig. 5 and 6 were generated by the same process can be rejected with very high confidence. This conclusion would be unaffected by moderate changes to the positions of the boundaries of regions 1 to 6 .

\section{Concluding Remarks}

The results of this experiment are consistent with the view that if the preconceived rules-of-thumb with which players may originally approach a game are not too firmly established, then they can be displaced by more sophisticated rules that take better account of the strategic realities of the situation. Moreover, there is evidence that subjects are willing to justify their new behavior by asserting that it is fair. It is not argued that these conclusions support the view that fairness/focal theories are mistaken. Nor is it claimed that subjects are natural gamesmen. Our belief is that a more sophisticated type of theory than either of these alternatives is necessary.

We do not believe that people are natural gamesmen, if the term "natu- 
ral gamesman" is taken to mean an individual who is familiar with all the results of game theory, who is capable of lightning mental calculations of great complexity, and who takes for granted that other individuals selected at random from the population at large have the same characteristics as themselves. In so far as people behave like gamesmen, it is presumably because they are capable, to some extent, of adapting their behavior to new situations. We do not believe that the manner in which people learn in game-like situations is optimal or even close to optimal. It is clearly often a hit-and-miss affair which operates below the level of consciousness to a considerable degree. Nevertheless, we believe that game theory can be useful in predicting the outcome of such learning processes.

One may speculate that people are equipped with rules-of-thumb that they use to settle conflicts of interest that arise in real-life bargaining situations and elsewhere, and that these rules-of-thumb embody fairness criteria or depend in other ways on salient or focal features of the environment in which they are used. We shall follow Dawkins (1976) in referring to such rules-of-thumb as memes. It seems unlikely that people think very hard about these memes when using them in the real-life situations to which they are adapted. One tends to question ingrained habits or customs only when their use generates unsatisfactory results.

What triggers a switch from one meme to another? We have no general theory to propose. Certainly, this experiment was not designed to answer such a question. Its design specifically excludes the rich variety of conversational or contextual cues that presumably prompt the substitution of one meme for another under the conditions of everyday life. At best, the experiment only serves to demonstrate that such switches from one meme to another can occur, and that game theory can be relevant in predicting the nature of the switch. There are evolutionary reasons why this may be thought plausible. One may ask: given a meme which is established in a human population, how does it manage to survive? Why does it not get displaced by an alternative meme? The game theorist's answer is that it survives because it is adapted to the environment in which it is commonly used. That is to say, it prescribes behavior that is in equilibrium. ${ }^{16}$ People will not usually be conscious of this fact and may be quite truthful in reporting that they are unmotivated by strategic considerations. But it is not necessary for individuals to know why a particular meme survives in order for it to survive.

If this view is correct, a least in some circumstances, then one should

\footnotetext{
${ }^{16}$ It goes without saying that this is a gross simplification. One must take into account the complexity of the meme's environment. The more complex the environment, the more difficult it will be for better adapted memes to surface, and the longer it will take for them to become established. One must also consider the cost to individuals of implementing complicated strategies. And so on.
} 
expcct to obscrve memes in operation that are triggered by hints or cues in the environment that match the strategic realities of the situation. We hope that the current paper will be seen as a confirmation of the viability of such a standpoint rather than as just a refutation of a naive version of the fairness/focal explanation of human behavior. Subjects were put in situations for which life did not seem to have equipped them in advance with a strongly established rule-of-thumb. Behavior then evolved which correlated with the strategic situation, and many subjects seemingly developed attitudes toward fairness that allowed them to rationalize this behavior in terms that were familiar to them.

This behavior of our subjects is clearly not supportive of those who might wish to argue that "fairness memes" are altogether irrelevant in describing the behavior of subjects in such laboratory experiments as ours. Moreover, we do not doubt that it is possible to construct experiments in which adaptation to the environment of the kind we observed does not occur, even with large incentives and long time spans for learning. One might frame the experiment in such a way as to trigger a form of the fairness meme that is very firmly established for use in a particular real-world context but which bears only a surface resemblance to the problem faced in the laboratory. ${ }^{17}$ Alternatively, it would not be hard to interfere with the learning process by confusing the issues facing the subjects. Indeed, we seem to have done so inadvertently under regimes 0 and 2 with the $£ 5$ cake by introducing a round number focal point at $£ 3.00$. Such experiments, confirming that fairness is relevant to the way people resolve bargaining problems, would not and do not refute the view that we are defending here.

In summary, in defending the relevance of game theory to actual bargaining behavior, we are not denying that fairness/focal theories are also relevant. We deny only that a theory of this type that ignores strategic considerations is likely to get to what lies at the heart of human bargaining behavior. Most of all, we want to emphasize the importance of learning and adaptation in this context.

\section{APPENDIX A: THE INSTRUCTIONS}

Different written instructions were offered to subjects depending on the regime of the game they were to play, and whether they would be player I or player II in their first bargaining session. The instructions below are for a subject about to begin as player I under regime 1 . The instructions for a

\footnotetext{
${ }^{17}$ Invoking the meme in such pathological circumstances is, of course, precisely what one would wish to do if one's aim were to study the mechanics of a particular established meme.
} 
subject about to begin as player $I$ under regime 3 were identical with the exception of the fourth paragraph. This was replaced, under regime 3, by the paragraph quoted in Section 3. Instructions for regimes 0 and 2 were the same except that the figure of $£ 3.20$ was replaced by $£ 1.80$. The necessary modifications for a subject about to begin as player II will be evident.

\section{Bargaining Experiment: Regime 1, Subject 0}

This is an experiment in which you will bargain via the computing equipment in front of you with the persons in the other booths. There will be ten separate and distinct bargaining sessions after each of which the person with whom you are paired may change. The initial six sessions are for practice. The remaining four sessions are "for real". The other four persons were recruited in the same way as you and the order in which you and they will be paired has been chosen at random.

In each of the ten sessions a "cake" which is always nominally worth $£ 5$ will be available for the two bargainers to share, provided they can come to an agreement on how it should be split. You and the other person will alternate in making proposals until either a proposal is accepted or the negotiations break down. You will make the first proposal in the first session but in five of the ten sessions it will be the other person who makes the first proposal.

Once a proposal is accepted, each of the two parties to the agreement will have his or her agreed share credited to their accounts. BUT, if the negotiations get broken off, the opportunity to split the cake will disappear. Instead, each player will receive a BREAKDOWN payment. In the first session, your breakdown payment is $20 \mathrm{p}$ and the other person's is $\$ 3.20$. But in five of the ten sessions, it will be your breakdown payment which is $£ 3.20$ and the other person's which is $20 \mathrm{p}$.

Breakdowns can only occur immediately after a proposal has been refused. It is the person who just refused a proposal who decides whether or not to break off the negotiations. If the decision is to continue bargaining, the cake SHRINKS TO $90 \%$ OF ITS PREVIOUS SIZE and so, of course, do the breakdown payments.

The final four sessions are for "real" and we will pay you all the money you make in these sessions immediately after the last bargaining session in which you are involved. The preceding six sessions are for practice only. In these sessions you will have to pretend that you are bargaining over real money.

In summary, you have to remember that each proposal which is made may be the last. But even if the negotiations get broken off, the session will not be a complete wash-out for you since you will still get your breakdown payment.

This is not an experiment to find out what sort of person you are. When we see the results, we shall neither know nor care who did what. We are only interested in what happens on average. So please do not feel that some particular kind of behavior is expected of you.

Before the first bargaining session, there will be a demonstration of the computing equipment. The demonstration program is started by pressing the SPACE BAR. But there is no need to hurry. You may have to wait for the other persons to be ready anyway. Read the instructions again if you think this may be helpful, or call the assistant if you need a question answered. 


\section{APPENDIX B: THE QUESTIONNAIRE}

It would help us if you would give brief answers to the following questions:

1 . Where were you recruited?

2. Were the instructions and the demonstration program clear?

3 . Were there any hitches during the experiment?

4. Why did you bargain the way you did?

5. On the scale below, indicate what you feel would be a fair way to split the cake if person 1's and person 2's breakdown payments were both $£ 0.20$.

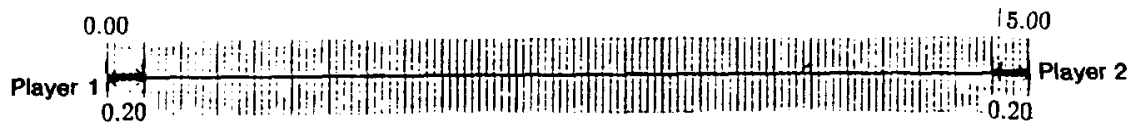

6. On the scale below, indicate what you feel would be a fair way to split the cake if person l's breakdown payment were $£ 0.20$ and person 2's breakdown payment were $£ 1.80$.

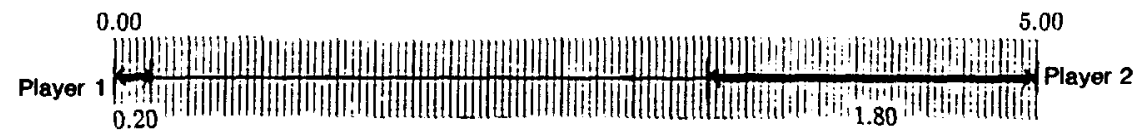

7. On the scale below, indicate what you feel would be a fair way to split the cake if person 1's breakdown payment were $£ 0.20$ and person 2 's breakdown payment were $£ 3.20$.

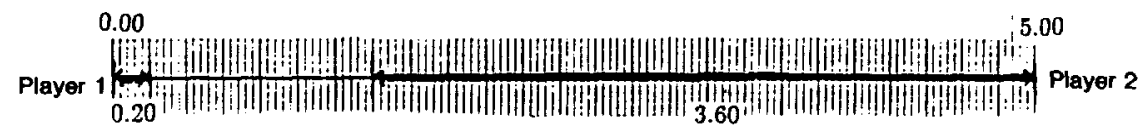

8. Is this the sort of situation in which people ought to "play fair" or is it socially acceptable for them to make what use they can of whatever bargaining power they have?

9. Would you bargain in the same way if you were put in the same situation tomorrow?

\section{appendiX C: The Monte Carlo Study}

The nonparametric test statistics used were the Kolmogorov-Smirnov (KS), Cramèr-von Mises (CM), Anderson-Darling (AD), and Epps-Singleton (ES) statistics. Let $F_{1}(x)$ and $F_{2}(x)$ denote two cumulative density functions (cdf's) defined on an observation space $X \in \mathbb{R}$. The null hypothesis is $H_{0}: F_{1} \equiv F_{2}$. The alternative, $H_{\mathrm{A}}$, is that $H_{0}$ is false. Let $\hat{F}_{1}$ and $\hat{F}_{2}$ denote the empirical cdf's generated by two independent random samples, of sizes $n_{1}$ and $n_{2}$, respectively, from $X$. Let $\hat{F}_{12}$ denote the empirical cdf generated by the combined sample, of size $n_{1}+n_{2}$. The KS, CM, and AD test statistics compare the empirical cdfs $\hat{F}_{1}, \hat{F}_{2}$, and $\hat{F}_{12}$. They are defined as follows: 


$$
\begin{aligned}
\mathrm{KS} & \equiv \sup _{x \in X}\left|\hat{F}_{1}(x)-\hat{F}_{2}(x)\right| \\
\mathrm{CM} & \equiv\left(\frac{1}{n_{1}}+\frac{1}{n_{2}}\right) \int_{X}\left(\hat{F}_{1}(x)-\hat{F}_{2}(x)\right)^{2} d F_{12}(x)
\end{aligned}
$$

and

$$
\mathrm{AD} \equiv\left(\frac{1}{n_{1}}+\frac{1}{n_{2}}\right) \int_{X} \frac{\left(\hat{F}_{1}(x)-\hat{F}_{2}(x)\right)^{2}}{\hat{F}_{12}(x)\left(1-\hat{F}_{12}(x)\right)}
$$

The ES statistic measures the distance between the empirical characteristic functions generated by the two samples. For an arbitrary but fixed integer $J \geq 1$ let $\left\{t_{1}, \ldots, t_{J}\right\}$ be real and positive numbers. Let $X_{i j}$ denote observation $i$ from sample $j$. The empirical characteristic function for sample $j$ is

$\phi_{j}\left(t_{k}\right) \equiv \frac{1}{n_{j}} \sum_{i=1}^{n_{j}}\left(\cos t_{k} X_{i j}+i \sin t_{k} X_{i j}\right)$

for $k=1, \ldots, J$ and for $j=1,2$.

Denote the $2 J \times n_{j}$ matrix of the real and imaginary parts of $\phi_{j}$ by

$$
G_{j}=\left(\begin{array}{cccc}
\cos t_{1} X_{1 j} & \cos t_{1} X_{2 j} & \cdots & \cos t_{1} X_{n_{j}} \\
\sin t_{1} X_{1 j} & \sin t_{1} X_{2 j} & \cdots & \sin t_{1} X_{n_{j} j} \\
\vdots & \vdots & & \vdots \\
\cos t_{J} X_{1 j} & \cos t_{J} X_{2 j} & \cdots & \cos t_{j} X_{n_{j} j} \\
\sin t_{J} X_{1 j} & \sin t_{J} X_{2 j} & \cdots & \sin t_{J} X_{n_{j} j}
\end{array}\right) \quad \text { for } j=1,2
$$

The average row sums of $G_{j}$ are denoted by the $2 J \times 1$ vector

$g_{j}=\frac{1}{n_{j}}\left(\sum_{i=1}^{n_{j}} \cos t_{1} X_{i j}, \sum_{i=1}^{n_{i}} \sin t_{1} X_{i j}, \ldots, \sum_{i=1}^{n_{j}} \cos t_{J} X_{i j}, \sum_{i=1}^{n_{i}} \sin t_{J} X_{i j}\right)^{T}$,

$$
\text { for } j=1,2 \text {. }
$$

The Epps-Singleton statistic is

$$
\mathrm{ES} \equiv c \cdot \frac{2 n_{1} n_{2}}{n_{1}+n_{2}}\left(g_{1}-g_{2}\right)^{T}\left(\hat{S}_{1}+\hat{S}_{2}\right)^{-1}\left(g_{1}-g_{2}\right),
$$

where $\hat{S}_{1}$ and $\hat{S}_{2}$ are the variance-covariance matrices of $G_{1}$ and $G_{2}$, respectively. $c$ is a small sample correction factor. The ES statistic is 


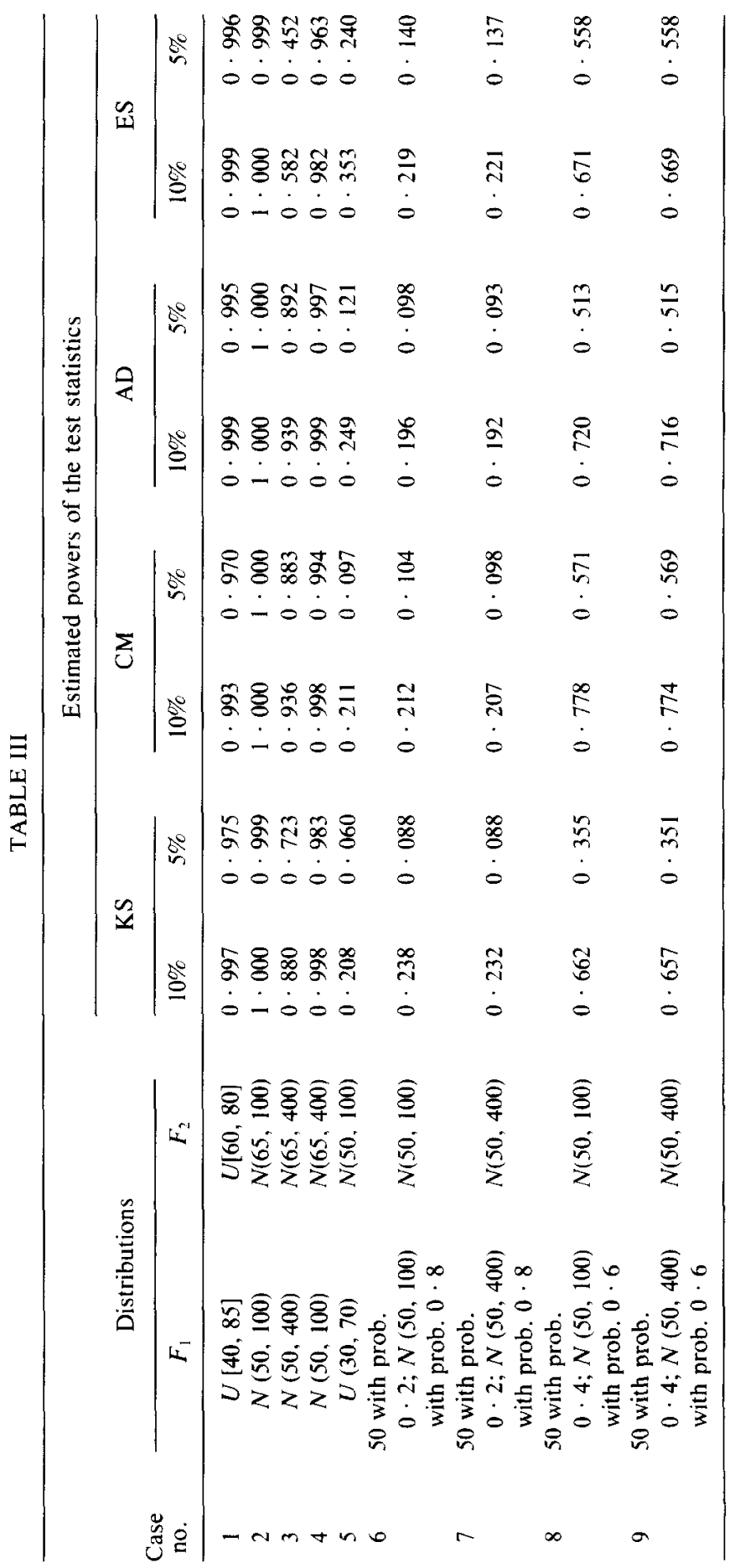


asymptotically distributed as a $\chi^{2}$ variable with $2 J$ degrecs of frecdom. We chose sample sizes of $n_{1}=n_{2}=40$ for our study since the sizes of our data sets are close to these values. Following the recommendations given in Epps and Singleton (1986) for such sample sizes, we chose $J=5,\left(t_{1}, t_{2}\right.$, $\left.t_{3}, t_{4}, t_{5}\right)=(0 \cdot 4,0 \cdot 8,1 \cdot 2,1 \cdot 6,2 \cdot 0)$ and $c=0 \cdot 849$.

The alternative hypotheses used in our study are listed in Table III. The table also presents the estimated powers of each test, for significance levels of 10 and $5 \% .10,000$ independent pairs of samples of 40 data were generated for each case and the power estimates for the 10 and $5 \%$ significance levels were computed as the fraction of the 10,000 replications for which the null hypothesis that $F_{1} \equiv F_{2}$ was rejected at these significance levels.

The table suggests that no test dominates the others at the $10 \%$ level. The KS test never wins at the 5\% significance level. The $\mathrm{CM}$ and $\mathrm{AD}$ tests generally do better than the KS and ES tests at either significance level for cases 2,3 , and 4 , which present cases of shifts of central location and/or variance. The CM test does best at recognizing differences in distribution due to the existence of a substantial mass point. (The ES test is nonparametric even for discontinuous cdfs. However, the KS, CM, and AD tests assume continuous cdf's and so are not truly nonparametric for cases 6 to 9.) The above results contrast with those obtained by Forsythe et al. who selected the AD and ES tests over the others on the basis of a study using samples of size 25 . Our study's results left us unable to discard any of the four tests.

\section{REFERENCES}

Binmore, K., Morgan, P., Shaked, A., and Sutton, J. (1989a). “Do People Exploit Their Bargaining Power? An Experimental Study," CREST Working Paper 89-15, University of Michigan.

Binmore, K., Osborne, M., AND Rubinstein, A. (1989b). "Noncooperative Models of Bargaining," CREST Working Paper 89-26, University of Michigan; in The Handbook of Game Theory, in press.

Binmore, K., Shaked, A., And Sutton. J. (1989c). "An Outside Option Experiment," Quart. J. Econ. 104, 753-70.

Binmore, K., Shaked, A., And Sutton, J. (1985). "Testing Noncooperative Bargaining Theory: A Preliminary Study," Amer. Econ. Rev. 75, 1178-1180.

Binmore, K., Shaked, A., and Sutton, J. (1988). "A Further Test of Noncooperative Bargaining Theory: Reply," Amer. Econ. Rev. 78, 837-839.

Cramton, P. (1988). "Dynamic Bargaining with Outside Options," Unpublished manuscript, Yale School of Management.

Dawkins, R. (1976). The Selfish Gene. Oxford: Oxford Univ. Press.

EpPs, T. W., And Singleton, K. J. (1986). "An Omnibus Test for the Two-Sample Problem Using the Empirical Characteristic Function," J. Statist. Comput. Simul. 26, 177203. 
Forsythe, R., Horowitz, J. L. Savin, N. E., and Sefton, M. (1988). "Replicability Fairness and Pay in Experiments with Simple Bargaining Games," Department of Economics, University of Iowa, Working Paper 88-03.

Friedman, J. H., AND RafSKy, L. C. (1979). "Multivariate Generalizations of the WaldWolfowitz and Smirnov Two-Sample Tests," Ann. Statist. 7, 697-717.

GÜth, W. (1990). "Behavioral Theory of Distributive Justice," in Applied Behavioral Economics (S. Maital, Ed.). Sussex: Brighton, in press.

Kahneman, D., Knetsch, J., and Thaler, J. (1986). "Fairness and the Assumptions of Economics," J. Bus. 59, 285-300.

Leventhal, G. (1980). "What Should Be Done with Equity Theory? New Approaches to the Study of Fairness in Social Relationships," in Social Exchange: Advances in Theory and Research (K. Gergen, M. Greenberg, and R. Willis, Eds.). New York: Plenum.

RoTh, A. (1985). "Towards a Focal Point Theory of Bargaining," in Game-Theoretic Models of Bargaining (A. Roth, Ed.). Cambridge: Cambridge Univ. Press.

Rubinstein, A. (1982). "Perfect Equilibrium in a Bargaining Model," Econometrica 50, 97-109.

Selten, R. (1978). "The Equity Principle in Economic Behavior." in Decision Theory and Social Ethics, Issues in Social Choice (H. Gottinger and W. Leinfellner, Eds.). Dordrecht: Reidel.

ShaKed, A. (1987). "Opting Out: Bazaars versus 'Hi Tech' Markets," STICERD Discussion Paper TE 87/159, London School of Economics.

Sutton, J. (1986). "Noncooperative Bargaining Theory-An Introduction," Rev. Econ. Stud. 53, 709-24.

Thibaut, J. (1968). "The Development of Contractual Norms in Bargaining: Replication and Variation," J. Conflict Resolution 12, 102-112.

Walster, E., Berscheid, E., ANd Walster, G. (1973). "New Directions in Equity Research," J. Personality Soc. Psychol. 25, 151-176. 